\title{
El síndrome de QT largo en anestesia. Una revisión a partir de un caso clínico
}

\author{
SEBASTIÁN CÓRDOVA V. ${ }^{1}$, SEBASTIÁN MELA S. ${ }^{2}$, CRISTIÁN GRESA B. ${ }^{3}$
}

\begin{abstract}
Becado Anestesiología y Reanimación, Universidad de Valparaíso.
Becado Anestesiología y Reanimación, Universidad Andrés Bello.

Anestesiólogo, Universidad de Valparaíso.

Centro de trabajo: Hospital Dr. Gustavo Fricke, Viña del Mar. Chile.
\end{abstract}

Se presenta el caso de una adolescente de 14 años que se programa en el Hospital Carlos Van Buren de Valparaíso para un retiro de material de osteosíntesis del brazo por una fractura antigua. $\mathrm{Su}$ evaluación preoperatoria no es particularmente inquietante. Se observa como una joven con una vida diaria normal. Su examen físico es normal. Presenta una historia de alergia a la aspirina y al metamizol. Como dato relevante evidencia consultas previas por episodios de palpitaciones autolimitadas y sin repercusiones. Fue referida por esta razón a consulta cardiológica, donde se le realizó un ecocardiograma informado como normal y un Test de Holter de arritmias.

El informe de éste último destaca: Conducción AV 1:1 con PR de 0,16 a 0,19 segundos (límite máximo para la edad). Conducción intraventricular impresiona normal (QRS 0,07 a 0,08 segundos). QTc estimado variable, desde 0,42 a más de 0,50 segundos. Conclusión: QTc variable. Muy probable Síndrome de QT largo. Extrasístoles ventriculares monomorfos aislados muy escasos". El cardiólogo inicia atenolol 25 mg cada 12 horas.

Basado en estos antecedentes, se planifica una anestesia total intravenosa con TCI de propofol y remifentanil. La paciente llega a pabellón premedicada con midazolam 7,5 mgr vo.

Se realiza preoxigenación con $\mathrm{FiO}_{2}$ 100\% 10 lt durante 3 minutos, monitorización con Presión arterial no invasiva, Electrocardiograma 5 deri- vadas, Oximetria de pulso y Capnografía.

Se procede a la inducción con TIVA (Remifentanil TCI $=4(0,2$ gamma $/ \mathrm{kg} / \mathrm{min})$ y Propofol TCI 3,5), se inserta Máscara Laringea proseal \# 3 acorde al peso (no se utilizaron bloqueadores neuromusculares para el procedimiento). Para la profilaxis de náuseas y vómitos se administró betametasona $8 \mathrm{mg}$ (el hospital no contaba con dexametasona en ese momento).

Se desarrolla procedimiento sin incidentes, Electrocardiograma sin alteraciones del QT ni alteraciones del Ritmo. Se decide despertar con infusión remifentanil $\mathrm{TCI}=1,5$ para disminuir la respuesta adrenérgica.

En las indicaciones postoperatorias:

- Manejo analgésico postoperatorio con Ketoprofeno $100 \mathrm{mgr}$ cada $8 \mathrm{~h} \mathrm{ev}+$ Paracetamol $500 \mathrm{mgr}$ cada $6 \mathrm{~h}$ vo y Morfina $2 \mathrm{mgr}$ ev de rescate.

- Quedó expresamente manifiesto no administrar droperidol (El hospital tampoco contaba con ondansetrón $\mathrm{u}$ otro antagonista $5-\mathrm{HT}_{3}$ ).

Los anestesiólogos con relativa frecuencia nos vemos enfrentados al concepto "QT largo". Es conocimiento generalizado que ciertas drogas utilizadas en la práctica anestésica, sobretodo en el área del manejo de las náuseas y vómitos postoperatorios, tienen repercusión sobre el intervalo QT del electrocardiograma. Otras condiciones patológicas como la hipokalemia y la hi-

Correspondencia a:

Sebastián Mela Sfeir

smela.sfeir@gmail.com 
pomagnesemia tienen un efecto similar. También hay una reconocida asociación entre el intervalo QT largo y el riesgo de presentar arritmias que pueden comprometer la hemodinamia e incluso la vida de los pacientes. Sin embargo, en ocasiones puede aparecer un caso como el descrito, un paciente que de base tenga una prolongación del intervalo QT. La pregunta a plantearse entonces es ¿cómo enfocamos el manejo perioperatorio de estos pacientes?. ¿Qué sabemos del intervalo QT y del síndrome de QT largo?. ¿Son éstas y otras drogas efectivamente peligrosas en esta condición?.

Esta revisión pretende entregar luces respecto a la fisiología y fisiopatología del intervalo QT, cómo se clasifican y manejan los distintos tipos de síndrome de QT largo y finalmente recomendaciones basadas en la evidencia disponible sobre el manejo perioperatorio de estos pacientes, poniendo énfasis en el efecto sobre éste que tienen distintas drogas anestésicas de uso habitual.

\section{EI Intervalo QT}

En el electrocardiograma, el intervalo QT corresponde al tiempo para la total depolarización y repolarización del ventrículo, es decir, desde el inicio de la Fase 0 hasta el fin de la Fase 3. Este se mide desde el inicio del complejo QRS hasta el final de la onda $\mathrm{T}$, habitualmente en las derivaciones DII, V2, V3 y V4, medido como el promedio entre 3 y 5 complejos consecutivos. En general, más que la derivación, es el intervalo QT más largo, o aquél donde se aprecia mejor el final de la onda $T$ en cualquiera de éstas, el que se toma como referente ${ }^{1,2}$.

En general se acepta que la duración del intervalo QT normal fluctúa entre los $390 \mathrm{~ms}$ y los $440 \mathrm{~ms}$ en adultos, existiendo debate sobre estos límites, encontrándose valores de $380 \mathrm{~ms}$ y $450 \mathrm{~ms}$ para cada extremo respectivamente ${ }^{3}$. La duración del intervalo se ve afectada por variables como la edad, sexo, peso corporal, tono simpáticovagal e incluso ritmo circadiano. Hay acuerdo en que el intervalo QT es unos $10 \mathrm{~ms}$ más largo en las mujeres en comparación con los hombres, debido a un acortamiento de este por efecto de la testosterona en la adolescencia, en el género masculino. Esta diferencia disminuye con la edad, pero se acepta un valor normal límite de
$460 \mathrm{~ms}$ en mujeres adultas. La obesidad también se ha asociado a mayor duración del intervalo ${ }^{4-6}$.

Existen menos estudios en la población pediátrica, aunque se acepta un intervalo QT hasta $440 \mathrm{~ms}$ como normal, y entre $440 \mathrm{~ms}$ y $460 \mathrm{~ms}$ como borderline $\mathrm{e}^{7,8}$.

La técnica empleada para su medición también influye de manera importante en la interpretación del intervalo QT, existiendo diferencias en los valores entre las distintas derivaciones ${ }^{9}$, velocidad de registro ${ }^{10}$, experiencia del observador y sistema de interpretación, ya sea manual o automatizado ${ }^{11}$.

Sin embargo, quizás el factor más importante para la correcta interpretación del intervalo QT es su relación inversamente proporcional con la frecuencia cardíaca. Debido a esta variabilidad, es necesario corregir el intervalo QT, normalizándolo para una frecuencia de 60 latidos por minuto. El cálculo del intervalo QT corregido (QTc) se hace de manera tradicional utilizando la fórmula de Bazett (QTc $=\mathrm{QT} / \sqrt{ } \mathrm{RR})$, que data de 1920. Se ha visto que esta fórmula sobrecorrige el valor del QT, especialmente a altas frecuencias, encontrando intervalos QT patológicamente largos hasta en un 30\% de ECG normales si se toma como corte un valor de $440 \mathrm{~ms}$, comparado con otras fórmulas menos utilizadas como la de Fridericia $(\mathrm{QTc}=\mathrm{QT} / \sqrt{ } \sqrt{\mathrm{RR}})$, la de Hodges $(\mathrm{QTc}=\mathrm{QT}+1,75$ [FC-60]) y la de Framingham $(\mathrm{QTc}=\mathrm{QT}+0,154[1-\mathrm{RR}])^{12}$. Nuevas fórmulas en desarrollo apuntan a estimar el intervalo QT de una manera no dependiente de la frecuencia cardíaca ${ }^{13}$.

Como puede verse, no hay un total consenso en cuanto al rango de valores considerados como normales para el intervalo QT. A pesar de esto, la mayoría de la evidencia acepta:

- <440 ms como normal.

- 440 - 460 ms como límite.

- >460 ms como prolongado o como probablemente patológico.

- $>500 \mathrm{~ms}$ como categóricamente patológico.

\section{La Dispersión de la Repolarización Transmural y la Arritmogénesis}

Se han identificado tres capas histológicas en el miocardio humano. El endocardio, el miocardio medio y el epicardio. Cada una cuenta con 


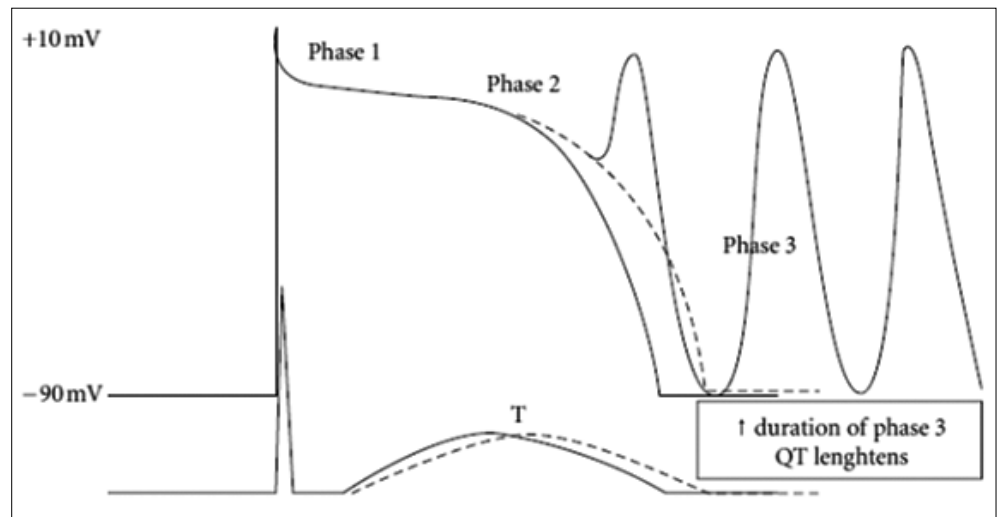

Figura 1. Postpotenciales tempranos sobre la Fase 3 pueden gatillar extrasístoles y eventualmente una arritmia ventricular. Kallergis EM, Goudis CA, Simantirakis EN, Kochiadakis GE, Vardas PE. Mechanisms, risk factors, and management of acquired long QT syndrome: a comprehensive review. The Scientific World Journal 2012; 2012:212178. Epub 2012/05/18.

células que difieren entre sí en sus propiedades electrofisiológicas, destacando las células del miocardio medio (Células M), las cuales poseen una repolarización más lenta en comparación con el resto de las capas. Este fenómeno se explicaría por una menor concentración o actividad de los canales $\mathrm{I}_{\mathrm{KS}}$ especialmente. De este modo, se produce una heterogeneidad espacial entre las distintas velocidades de repolarización en el miocardio, lo que se conoce como dispersión de la repolarización transmural (DRT). En el electrocardiograma, la repolarización ventricular se manifiesta en forma de la onda $\mathrm{T}$, y la forma de ésta sería atribuible a la DRT. Esta situación normal es la que, reuniéndose una serie de condiciones, podría generar el sustrato para la aparición de arritmias ventriculares potencialmente letales ${ }^{14}$.

La prolongación del intervalo QT por diferentes causas puede teóricamente aumentar la DRT. Como resultado, el miocardio demora más en llegar a su estado de reposo eléctrico en todo su espesor, debido a que mientras hay zonas en la pared que ya están listas para una nueva depolarización (Fase 4), otras aún se encuentran en la fase de repolarización (Fase 3). Estas últimas, por estar en su período refractario relativo, se encuentran vulnerables a la aparición de postpotenciales tempranos, los que pueden generar episodios de R sobre T, depolarizando la célula y gatillando una arritmia ventricular polimorfa como la Torsades de Pointes (TdP) (Figura 1). Esta habitualmente es autolimitada, pero puede perpetuarse por un mecanismo de reentrada y degenerar en una fibrilación ventricular. Naturalmente, mientras mayor sea la DRT, mayor es la probabilidad de que se produzca este fenómeno en algún punto del miocardio.

Sin embargo, la prolongación del intervalo QT per se no necesariamente aumenta la DRT, como se ha evidenciado en drogas como pentobarbital y amiodarona, que reconocidamente prolongan el intervalo ${ }^{15,16}$. Por esta razón, encontrar un QT largo en un ECG tampoco necesariamente es sinónimo de riesgo arritmogénico. Debido a esto se han propuesto distintas formas de estimar la DRT. La más sencilla es la medición de la dispersión del QT (QTd), que no es más que la diferencia entre el QT más largo y el QT más corto medido en las distintas derivaciones. La medición del QTd no ha mostrado ser un indicador efectivo de arritmogenicidad, excepto en valores groseramente elevados (más de 100 $\mathrm{ms}$ ), por lo que su utilidad es discutible ${ }^{17-19}$. Otro indicador más prometedor parece ser el intervalo Tpeak-end (Tp-e), el cual se mide desde el punto más alto de la onda $\mathrm{T}$ hasta su final en la línea isoeléctrica. El punto más alto de T correspondería a la repolarización más temprana en el epicardio, mientras que el fin de T correspondería a la repolarización final del miocardio medio. La prolongación del Tp-e parece relacionarse mejor 
con la DRT real ${ }^{20-22}$. El cociente Tp-e/QT también parece ser buen predictor de arritmogénesis ${ }^{23}$. Se han propuesto otros indicadores de inestabilidad eléctrica basados en la morfología y variabilidad de la onda T, sobretodo en casos de QT largos congénitos ${ }^{24}$.

\section{EI Síndrome de QT largo}

Una vez planteada la sospecha electrocardiográfica de intervalo QT largo, debemos analizar la causa subyacente. El síndrome de QT largo (SQTL o LQTS) está constituido por una serie de patologías distintas, con distintas etiologías, las cuales comparten la alteración en el ECG pero plantean diferentes abordajes terapéuticos. En general, los podemos clasificar a grandes rasgos en LQTS congénito y LQTS adquirido.

\section{Síndrome de QT largo congénito}

El Síndrome de QT largo congénito (cLQTS) obedece a una serie de anomalías en la repolarización ventricular transmitidas genéticamente. Estos pacientes habitualmente tienen una historia de eventos sincopales, palpitaciones e incluso paro cardiorrespiratorio recuperado, pero en ocasiones pueden ser asintomáticos. Antes del desarrollo de estudios genéticos para el diagnóstico de enfermedades se habían identificado principalmente dos variantes de esta enfermedad. El síndrome de Romano-Ward consiste en intervalo QT largo y alteraciones en la morfología de la onda $\mathrm{T}$ asociadas a episodios sincopales atribuibles a Torsade des pointes y muerte súbita en niños. Se transmite de manera autosómica dominante y tiene una prevalencia estimada en 1:3.000 personas. Típicamente, los episodios se manifiestan en relación a stress físico, emociones intensas e incluso ruidos fuertes, aunque algunos enfermos presentan manifestaciones clínicas en reposo e incluso durante el sueño. Se han descrito pacientes con intervalos QT en rangos límite o incluso normales (cLQTS “oculto"), lo que suele generar problemas en el diagnóstico, confundiéndolo con otras enfermedades como epilepsia o síncope vasovagal.

La otra variante menos común de cLQTS es el síndrome de Jervell Lange-Nielsen, el cual además de las alteraciones mencionadas, se acompaña de sordera congénita. Se transmite de manera autosómica recesiva. Su prevalencia estimada es de 1:200.000 y es particularmente alta en países como Noruega. Estos pacientes habitualmente tienen intervalos QT marcadamente prolongados $(>500 \mathrm{~ms})$, presentan síntomas a edades más tempranas y su riesgo de padecer arritmias y muerte súbita es mayor ${ }^{25,26}$.

Con el advenimiento del diagnóstico genético, a mediados de los años noventa se identificaron distintas mutaciones en los canales iónicos responsables del potencial de acción en el miocardio. Estas "canalopatías" finalmente explican las diferencias en la presentación del cLQTS dependiendo de cuál sea la mutación en particular. Algunas personas presentan más de una mutación, lo que traduce en una mayor severidad de la enfermedad. El efecto de estas mutaciones se traduce en la prolongación del potencial de acción del miocardiocito por distintos mecanismos, como el bloqueo o enlentecimiento de los eflujos de $\mathrm{K}+\mathrm{o}$ el aumento de función de canales de $\mathrm{Na}+$ y muy posiblemente de $\mathrm{Ca} 2+$.

Las primeras mutaciones identificadas fueron denominadas convenientemente LQT1, LQT2 y LQT3. Estas mutaciones comprenden hasta un $80 \%$ de los casos identificados, puesto que en la actualidad se han descrito hasta doce variantes, algunas en extremo raras (Tabla 1). LQT1 se presenta como mutaciones en los canales rectificadores $\mathrm{I}_{\mathrm{KS}}$. Este grupo presenta manifestaciones clínicas asociadas al ejercicio y stress emocional. LQT2 comprende mutaciones de los $\mathrm{I}_{\mathrm{KR}}$. Estos pacientes son susceptibles a estímulos auditivos intensos. LQT3 se asocia a mutaciones en los canales de sodio voltaje dependientes $\mathrm{I}_{\mathrm{Na}}$, otorgándoles una "ganancia en su función", manteniendo el ingreso de sodio a la célula por más tiempo, prolongando el potencial de acción. Este grupo en general puede manifestar episodios sincopales y muerte súbita en reposo o durante el sueño ${ }^{25-32}$. Todas las variantes de cLQTS presentan aumento de la DRT como factor fisiopatológico clave, independiente de la duración del intervalo QT.

Manejo de pacientes con cLQTS: consiste principalmente de tres pilares. Como ya se mencionó, la mayoría de estos enfermos son susceptibles a la actividad adrenérgica y estímulos intensos. Así, una de las primeras medidas terapéuticas es precisamente evitar este tipo de situa- 
ciones en el quehacer diario. Evitar deportes de alta exigencia o evitarlos del todo en casos más severos. Ambientes tranquilos, libres de ruidos molestos, ansiolisis por diversas vías. En otros casos debe evitarse la exaltación brusca como puede ser un despertar súbito, e incluso monitorización durante el descanso en enfermos con episodios asociados al sueño. Este manejo debe ser individualizado según el tipo de cLQTS en particular, siendo algunas intervenciones efectivas para algunos tipos e inefectivas o innecesarias para otros ${ }^{27}$.
La terapia antiadrenérgica es otro pilar fundamental en el manejo de estos enfermos. El uso de betabloqueadores está ampliamente aceptado por su efecto depresor de la velocidad de conducción $\mathrm{y}$ aumento del periodo refractario efectivo del miocardiocito. Ambos mecanismos previenen la aparición de fenómenos de reentrada. Propanolol es la elección más común. Sin embargo, el beneficio mayor de la terapia con betabloqueadores se observa en los enfermos afectados de LQT1 ${ }^{33}$ y en menor grado en LQT2. El efecto es mínimo en pacientes con LQT3 por ser otro canal ióni-

Tabla 1. Clasificación por genotipo del síndrome de QT largo congénito. Kaufman ES. Mechanisms and clinical management of inherited channelopathies: long QT syndrome, Brugada syndrome, catecholaminergic polymorphic ventricular tachycardia, and short QT syndrome. Heart rhythm : the official journal of the Heart Rhythm Society. 2009; 6(8 Suppl):S51-5. Epub 2009/08/06

\begin{tabular}{|c|c|c|c|}
\hline Tipo & Gen & Función & Frecuencia \\
\hline LQT1 & KCNQ1 & $\mathrm{I}_{\mathrm{KS}} \downarrow$ & $30-35 \%$ \\
\hline LQT2 & $\mathrm{KCNH} 2$ & $\mathrm{I}_{\mathrm{KR}} \downarrow$ & $25-30 \%$ \\
\hline LQT3 & SCN5A & $\mathrm{I}_{\mathrm{Na}} \uparrow$ & $5-10 \%$ \\
\hline LQT4 & ANK2 & $\mathrm{I}_{\mathrm{Na}, \mathrm{K}} \downarrow$ & $1-2 \%$ \\
\hline LQT5 & KCNE1 & $\mathrm{I}_{\mathrm{KS}} \downarrow$ & $1 \%$ \\
\hline LQT6 & KCNE2 & $\mathrm{I}_{\mathrm{KR}} \downarrow$ & RARO \\
\hline LQT7 & KCNJ2 & $\mathrm{I}_{\mathrm{K} 1} \downarrow$ & RARO \\
\hline LQT8 & CACNA1C & $\mathrm{I}_{\mathrm{Ca}-\mathrm{L}} \uparrow$ & RARO \\
\hline LQT9 & CAV3 & $\mathrm{I}_{\mathrm{Na}} \uparrow$ & RARO \\
\hline LQT10 & SCN4B & $\mathrm{I}_{\mathrm{Na}} \uparrow$ & RARO \\
\hline LQT11 & AKAP9 & $\mathrm{I}_{\mathrm{KS}} \downarrow$ & RARO \\
\hline LQT12 & SNTA1 & $\mathrm{I}_{\mathrm{Na}} \uparrow$ & RARO \\
\hline
\end{tabular}

Tabla 2. Caracterización clínica de los cLQTS más comunes. Owczuk R, Wujtewicz MA, Zienciuk-Krajka A, LasinskaKowara M, Piankowski A, Wujtewicz M. The influence of anesthesia on cardiac repolarization. Minerva anestesiologica. 2012;78(4):483-95. Epub 2012/02/10

\begin{tabular}{|c|c|c|c|}
\hline \multicolumn{4}{|c|}{ Características de los síndromes LQT1, LQT2 y LQT3 } \\
\hline & LQT1 & LQT2 & LQT3 \\
\hline Corriente anómala & $\mathrm{I}_{\mathrm{KS}}$ & $\mathrm{I}_{\mathrm{KR}}$ & $\mathrm{I}_{\mathrm{Na}}$ \\
\hline ECG & $\begin{array}{l}\text { - Onda T prolongada } \\
\text { - Patrón de T ancho } \\
\text { - Segmento ST corto }\end{array}$ & $\begin{array}{l}\text { - Onda T de baja amplitud } \\
\text { - Onda T bífida inferolateral }\end{array}$ & $\begin{array}{l}\text { - Segmento ST largo } \\
\text { - Onda T angosta }\end{array}$ \\
\hline Gatillantes de TdP & $\begin{array}{l}\text { - Ejercicio } \\
\text { - Sorpresa } \\
\text { - Rabia } \\
\text { - Miedo } \\
\text {-Estimulación adrenérgica }\end{array}$ & $\begin{array}{l}\text { - Estímulo auditivo } \\
\text { - Período postparto } \\
\text { - } 50 \% \text { en reposo o sueño }\end{array}$ & $\begin{array}{l}\text { - Principalmente en reposo o } \\
\text { sueño }\end{array}$ \\
\hline Eficacia del betabloqueo & +++ & ++ & \pm \\
\hline
\end{tabular}


co el afectado, pudiendo incluso ser deletéreo al aumentar la DRT en este subgrupo ${ }^{34-36}$. Teóricamente, estos pacientes pudieran beneficiarse con el uso de antiarrítmicos de clase I como flecainida y mexiletina, que bloquean los canales de sodio, aunque la evidencia aún es limitada ${ }^{37,38}$.

Por último, en los pacientes de mayor riesgo, que no responden bien al betabloqueo o que ya han tenido un episodio de paro cardiocirculatorio recuperado, se indican procedimientos más invasivos como la denervación cardiaca izquierda vía toracotomía, que consiste en la extirpación de los primeros cuatro ganglios torácicos ${ }^{39}$, y/o la implantación de un cardiodesfibrilador ${ }^{40-42}$. Estos procedimientos se realizan excepcionalmente en pacientes muy seleccionados

\section{Síndrome de QT largo adquirido}

Se habla de Síndrome de QT largo adquirido (aLQTS) en cualquier condición no congénita que se manifieste en forma de prolongación del Intervalo QT. A diferencia de los pacientes congénitos, la principal causa del aLQTS es la inducción por drogas. Se han identificado numerosas drogas con una reconocida capacidad de prolongar el QT, y con el tiempo van apareciendo nuevas asociaciones. El mecanismo principal de la prolongación es vía el bloqueo de la expresión de los canales Ikr, que son codificados por el gen hERG. Este bloqueo se manifiesta como prolongación de la Fase 3 del potencial de acción. Para un seguimiento más detallado de las drogas consideradas de riesgo, el lector puede referirse al sitio www.torsades.org

Además, existen otras situaciones que son consideradas factor de riesgo para padecer TdP en el contexto de un aLQTS por drogas, como lo son algunas cardiopatías estructurales o la inhibición del citocromo CYP3A4 por otra droga en uso, por lo que se considera que en estos pacientes el mayor riesgo arritmogénico estaría dado por la suma de factores presentes más que la sola presencia de la droga implicada en cada caso (Tablas 3 y 4). Por último, en contadas ocasiones, puede darse que un paciente tenga un sustrato genético, el cual se vea exacerbado por el uso

Tabla 3. Factores de riesgo identificados para la aparición de TdP asociado al uso de drogas que prolongan el intervalo QT. Kaye AD, Volpi-Abadie J, Bensler JM, Kaye AM, Díaz JH. QT interval abnormalities: risk factors and perioperative management in long QT syndromes and Torsades de Pointes. Journal of anesthesia. 2013;27(4):575-87. Epub 2013/02/16

\begin{tabular}{|l|}
\hline Factores de riesgo para LQTS inducido por drogas y Torsades de Pointes \\
Sexo femenino \\
Edad avanzada \\
Alteraciones electrolíticas: Hipokalemía, hipomagnesemia, hipocalcemia \\
Bradicardia \\
Bloqueo AV completo \\
Cardiopatía estructural: Infarto, Falla cardiaca, Miocardiopatía, Valvulopatía \\
Intervalo QT basal > $450 \mathrm{~ms}$ \\
Complejos ventriculares prematuros con secuencia corto-largo-corto \\
Onda T variable \\
Cardioversión reciente por fibrilación auricular \\
\hline Síndrome QT Largo Congénito subclínico \\
Polimorfismos genéticos de genes que codifican canales iónicos \\
\hline Altas dosis o concentraciones de drogas que prolongan el QT \\
Uso concomitante de droga que prolonga QT y otra que inhiba su metabolismo \\
Metabolizadores lentos \\
Falla hepática o renal \\
\hline Obesidad (asociación poco clara) \\
\hline
\end{tabular}


Tabla 4. Drogas inhibidoras del CYP3A4. Kaiiergis EM, Goudis CA, Simantirakis EN, Kochiadakis GE, Vardas PE. Mechanisms, risk factors, and management of acquired long QT syndrome: a comprehensive review. The Scientific WorldJournal. 2012:2012:212178. Epub 2012/05/18

\begin{tabular}{|l|}
\hline Inhibidores de CYP3A4 \\
Antihipertensivos \\
• Dihidralazina \\
- Diltiazem \\
• Verapamilo \\
Antidepresivos / Ansiolíticos \\
- Fluoxetina \\
• Midazolam \\
Antimicrobianos / Antivirales \\
• Macrólidos \\
- Isoniazida \\
- Terapia VIH \\
Endocrinológicos \\
- Anticonceptivos \\
• Etinilestradiol \\
- Antiprogestágenos \\
- Tamoxifeno \\
Constituyentes alimenticios \\
• Bergamotina (jugo de uva) \\
• Glabridina (regaliz) \\
\hline
\end{tabular}

de alguna droga potencialmente arritmogénica. Estos pacientes son considerados como cLQTS "frustros", donde una baja penetrancia impidió la manifestación completa del síndrome congénito, pero al parecer presentan algún grado de desorden electrofisiológico subyacente, el cual no se manifiesta hasta que se reúnen las condiciones necesarias ${ }^{30,43,44}$.

El manejo del aLQTS se basa en la discontinuación de la droga involucrada o bajar la dosis siempre y cuando sea posible, así como también otras drogas que interfieran con el metabolismo de la droga considerada responsable. A diferencia del cLQTS, los pacientes con aLQTS presentan especial riesgo en relación a alteraciones electrolíticas y aumento del tono vagal con bradicardia. Por esto, es especialmente importante corregir estas anomalías tan pronto como sea posible. La bradicardia predispone la aparición de extrasístoles que son seguidos de una pausa compensatoria. La aparición de un nuevo extrasístole en este contexto puede gatillar una TdP (secuencia corto-largo-corto). El uso de magnesio también se ha reconocido como útil en el manejo de estos pacientes debido a sus propiedades estabilizadoras de la membrana, posiblemente por bloqueo de corrientes de sodio y calcio, aunque éste per se no acorta el Intervalo QT. La utilización con éxito de antiarrítmicos como lidocaína y fenitoína se resume a algunos casos esporádicos. Por último, el uso de marcapasos transitorio o definitivo en frecuencias altas (overdrive pacing) logra acortar el QT en pacientes de alto riesgo $0^{45}$. El uso de isoproterenol permite el mismo efecto y se utiliza como terapia puente previo a una solución más definitiva ${ }^{43,46-49}$.

\section{EI Intervalo QT largo en anestesia}

Como se ha mencionado, no es del todo inusual encontrar una prolongación del intervalo QT en el paciente programado para cirugía. Numerosas variables fisiológicas y patológicas pueden contribuir a la prolongación de la repolarización ventricular. El anestesiólogo debe estar preparado para entender y enfrentar esta situación con el mínimo riesgo posible para el paciente. Una minuciosa evaluación preoperatoria debe alertarnos sobre la presencia de un LQTS. Antecedentes de palpitaciones, síncopes y paro cardiorespiratorio con explicación poco clara, en relación a estímulos puntuales como el ejercicio, emociones fuertes o sonidos estridentes, sobretodo en la población pediátrica, nos orientan a sospechar un cLQTS, recordando adicionalmente que estos pacientes pueden tener de base un intervalo QT normal, manifestando la anomalía en el perioperatorio ${ }^{50}$. El antecedente de sordera congénita o una historia familiar de eventos similares fortalecen aún más un posible diagnóstico. Por otro lado, el uso concomitante de drogas, alteraciones electrolíticas y cardiopatías orgánicas entre otros factores son más orientadores hacia un aLQTS. Puesto que el estudio y diagnóstico genético en los posibles sospechosos no corresponde a la realidad nacional (como tampoco lo es de rutina en países más desarrollados), la acuciosidad del clínico puede marcar la diferencia en estos casos y además solicitar una oportuna evaluación cardiológica previa para optimizar el manejo de estos enfermos. Exámenes preoperatorios y un ECG deben estar al día . Una adecuada comunicación entre los participantes del caso es clave. 
En la preparación de estos pacientes, debemos tener en consideración entonces la posible causa del LQTS. El manejo de los pacientes con cLQTS comprende la minimización de cualquier estímulo que pueda ser considerado peligroso. Un ambiente tranquilo y libre de ruidos estridentes es necesario. En el caso de los niños más pequeños, la compañía de los padres o alguna persona de su confianza ayuda a minimizar la angustia, pudiendo estar presentes hasta el inicio de la anestesia. La premedicación ha mostrado ser de utilidad en estos casos con el mismo fin. Midazolam es la droga de elección en estos casos al no tener mayor efecto sobre el QTc ${ }^{51}$. No hay mayor evidencia respecto a otras benzodiacepinas en este contexto. El uso de ketamina no es recomendable por su efecto simpáticoestimulante ${ }^{46,52,53}$. Para la instalación de vías venosas se recomienda usar anestesia tópica sobre el área, como la crema EMLA u otro preparado, o bien, realizar la punción una vez dormido el paciente. La temperatura del pabellón debe ser adecuada y se deben tomar activas medidas para evitar el enfriamiento del paciente, puesto que se ha visto que la hipotermia también causa prolongación del $\mathrm{QT}^{54,55}$, así como puede alterar el metabolismo de las drogas potencialmente peligrosas, manteniendo niveles plasmáticos mayores y por más tiempo. La corrección de cualquier anomalía electrolítica debe realizarse oportunamente, especialmente alteraciones del potasio, magnesio y calcio. Se ha mencionado también el uso de magnesio preoperatorio a dosis de $30 \mathrm{mg} / \mathrm{kg}$ para prevenir la aparición de TdP incluso con valores de magnesio normales. En caso de arritmias previas, casos confirmados, o elevada sospecha, es recomendable tener a mano un cardiodesfibrilador con función de marcapasos externo e incluso poner los parches en el paciente previo al inicio de la anestesia. Esto último es especialmente válido en los pacientes con tendencia a la bradicardia o han presentado arritmias pausa-dependientes. Como se mencionó anteriormente, el uso de isoproterenol puede ser útil mientras se define un manejo más definitivo, aunque debe recordarse que éste al tener un efecto adrenérgico está contraindicado en pacientes con cLQTS ${ }^{56}$. En caso de presentarse un paciente portador de un dispositivo tipo marcapasos o cardiodesfibrilador implantado, deben tomarse las precauciones para evitar descargas accidentales, pidiendo al cardiólogo previamente que suprima la función de desfibrilación. El uso de electrocauterio puede interferir también con el sensado del dispositivo, siendo recomendable también dejarlo en modo asincrónico previamente o bien teniendo a mano un imán en caso de necesidad. La instalación de la placa (electrodo de retorno) del electrocauterio, debe ser lo más lejos posible del marcapasos, con el fin de evitar que la corriente pase cerca o a través de él, pudiendo dañarlo. Por último, los pacientes utilizando betabloqueadores no deben suspender su tratamiento. Cualquier tratamiento médico de otra índole con drogas que pudieran tener efecto de prolongación del QT debe ser suspendida con tiempo o bajar su dosis al mínimo terapéutico de ser posible ${ }^{52,57}$.

Debe realizarse monitoreo continuo del intervalo QT, idealmente en II o precordiales V2$\mathrm{V} 4$, donde parece haber mejor correlación con el intervalo real. La morfología de la onda $\mathrm{T}$ proporciona información adicional. Algunos autores recomiendan monitorización invasiva con línea arterial y acceso venoso central, pensando en la posibilidad de instalar un marcapasos transvenoso. La decisión habitualmente descansa en el criterio y el análisis de cada caso en forma individual $^{46,58}$.

Para la inducción, es necesario procurar tanto una droga segura, como también asegurar una adecuada profundidad y simpáticolisis para el momento de la laringoscopía, uno de los mayores estímulos adrenérgicos a contrarrestar. Se ha reportado la utilidad de la lidocaína en este escenario $^{59}$. Se mencionó anteriormente que el pentobarbital tiene un efecto prolongador del intervalo QT, sin embargo, su efecto sobre la DRT es despreciable, por lo que su uso no estaría contraindicado $^{15}$. Propofol, por otro lado, no ha mostrado tener un efecto significativo sobre la repolarización ventricular, incluso corrigiendo la prolongación del QT inducida por sevoflurano, constituyéndose en la droga de elección para la inducción de la hipnosis ${ }^{60-64}$. Ketamina, por las razones expuestas anteriormente, no es recomendable. En relación al etomidato, existen reportes que lo catalogan como seguro, aunque no sería superior al propofol $1^{65,66}$.

El uso de opioides para la abolición del estímulo adrenérgico es fundamental. Distintos opioides han sido estudiados y en general se acepta que son seguros. Fentanyl, remifentanil 
y alfentanil no tienen mayor efecto sobre la repolarización ${ }^{67,68}$. Sufentanil por el contrario, sí prolonga el QT, aunque esto se basa en un reporte antiguo y en dosis altas ${ }^{69}$. Existe menos evidencia en cuanto a los alfa-2 agonistas como dexmedetomidina. Por su mecanismo de acción, es factible que la autosupresión en la liberación de noradrenalina presináptica tenga un efecto inhibidor contra arritmias supraventriculares y ventriculares, como se ha visto en modelos animales ${ }^{70}$. Sin embargo, hay reportes de marcada prolongación del intervalo QT asociado a su uso, atribuido a su efecto hipotensor y bradicardizan$t^{71}$. En la actualidad no hay recomendaciones sobre su perfil de seguridad en este escenario.

En cuanto a los relajantes neuromusculares, debemos elegir aquél que reúna el mejor perfil. No debe tener efecto autonómico marcado, del tipo bradicardia o taquicardia, ni producir alteraciones en el potasio. Es deseable que no libere histamina y que su acción sea breve y predecible para evitar el uso de agentes anticolinesterásicos en su reversión. El uso de succinilcolina no se recomienda en estos pacientes ${ }^{53,72}$. Atracurio y vecuronio no han mostrado tener efecto sobre el intervalo $\mathrm{QT}^{51}$. Por su efecto bradicardizante, teóricamente no es deseable utilizar agentes anticolinesterásicos, además su asociación con anticolinérgicos como la atropina se asocia a la aparición de taquicardia, la cual puede ser aún más deletérea ${ }^{73-77}$. Sugammadex, la más reciente droga utilizada en la reversión del bloqueo neuromuscular por rocuronio y vecuronio, ha mostrado no tener mayor efecto sobre el intervalo QT y ha sido utilizada con éxito en pacientes de riesgo $^{78-81}$.

Todos los agentes halogenados causan prolongación de la repolarización cardiaca en distintos grados, aunque su efecto arritmogénico es materia de mucha discusión ${ }^{82-84}$, encontrando frecuentemente resultados dispares. La DRT no parece verse afectada mayormente con estos agentes. Isoflurano parece ser la elección, habiendo varios reportes de su utilización sin resultados adversos. Sevoflurano prolonga el QT sin afectar mayormente la $\mathrm{DRT}^{62,85,86}$, aunque se han reportados arritmias ventriculares en relación a su uso en pacientes con factores de riesgo ${ }^{87}$. Desflurano prolonga el $\mathrm{QT}^{88}$, y en mayor grado que sevoflurano $^{61,89}$. Halotano no es recomendable por su efecto sensibilizador del miocardio a las cateco- laminas, aunque ha mostrado tanto prolongación como acortamiento del $\mathrm{QT}^{90}$. Su uso actual es muy limitado. En virtud de toda la información disponible y la falta de consenso, parece ser recomendable evitar el uso de halogenados si es factible, en favor de alternativas como TIVA o anestesia regional ${ }^{46,52,53,58,91}$.

La anestesia regional se basa en los mismos principios de manejo de este tipo de pacientes. Se ha visto que la anestesia espinal también prolonga el $\mathrm{QTc}^{92}$, aunque el bloqueo adrenérgico y la analgesia que provee son deseables. Existen reportes de su uso exitoso en pacientes con LQTS en el campo de la obstetricia ${ }^{93-96}$. Algunos autores reportan la anestesia epidural como alternativa más segura, aludiendo al hecho de una instauración más gradual, con menor hipotensión, y por tanto, menos probabilidad de uso de drogas simpaticomiméticas ${ }^{97}$. Existe poca evidencia disponible sobre el efecto de los anestésicos locales en el LQTS, aunque se ha reportado el uso de lidocaína, bupivacaína y levobupivacaína neuroaxial sin presentar inconvenientes ${ }^{93,95,98,99}$. En general, hay consenso en no utilizar epinefrina como complemento a la dosis neuroaxial. Específicamente en el área de la obstetricia, se ha visto que el uso de la oxitocina y análogos como carbetocina pueden prolongar el QTc ${ }^{100-102}$, aunque un estudio posterior propone como causa un mecanismo indirecto por cambios en el tono vasovagal atribuido a la hipotensión causada por estas drogas ${ }^{103}$.

Para el manejo de la hipotensión se recomienda utilizar agonistas alfa puros como fenilefrina, teniendo en consideración su efecto sobre la frecuencia cardiaca ${ }^{104}$. Durante la ventilación con presión positiva, es necesario evitar las maniobras que imiten una Valsalva, como reclutamiento alveolar o PEEP alto, puesto que también contribuyen a la prolongación del QTc ${ }^{105,106}$. El uso de neumoperitoneo en cirugía laparoscópica tendría el mismo efecto ${ }^{107}$.

La emergencia de la anestesia general debe ser igual de cuidadosa, puesto que constituye otro momento de stress adrenérgico importante, especialmente en niños. Puede ser de utilidad realizar una extubación profunda siempre que sea posible ${ }^{91}$.

El manejo postoperatorio de estos pacientes es igual de importante. Debe proporcionarse un ambiente tranquilo y temperado. El dolor posto- 
peratorio debe ser tratado en forma agresiva para evitar descargas adrenérgicas. Opioides de uso relativamente común en el postoperatorio como metadona y petidina son considerados no seguros, existiendo reportes de arritmias asociados a su uso en dosis elevadas o en casos de alteraciones genéticas no detectadas previamente ${ }^{108-111}$.

El manejo de las náuseas y vómitos postoperatorios puede ser problemático. Desde la aparición de la black box warning de la FDA en relación al uso de droperidol en 2001, su uso disminuyó sustancialmente. Sin embargo, esta recomendación ha sido continuamente cuestionada por muchos investigadores. Se ha visto que la prolongación del intervalo QT, así como el riesgo de arritmias por droperidol es dosis dependiente, muy por encima de las dosis comúnmente utilizadas para el manejo de la emesis postoperatoria. Más aún, la advertencia de la FDA se hizo en base a diez casos reportados de TdP, no logrando establecer el uso de droperidol como causa directa en ninguno de ellos ${ }^{112}$, por lo que muchos autores consideran la advertencia de la FDA exagerada o innecesaria, aludiendo también a la seguridad avalada por décadas de uso ${ }^{113}$. Se ha propuesto también la definición de un umbral terapéutico para su uso ${ }^{114}$. Además, hay varios estudios que comparan droperidol con los antagonistas 5-HT3, resultando tan efectivo como estos últimos, los cuales también han mostrado prolongar el QTc. Pese a que no son considerados como más seguros en este aspecto, comparados con droperidol, los antago- nistas 5-HT3 no presentan reparos por parte de la $\mathrm{FDA}^{115-119}$, y constituyen la primera línea de tratamiento para el manejo de la emesis postoperatoria según el último consenso ${ }^{120}$. La discusión se mantiene, aunque la recomendación general en el manejo de las náuseas y vómitos postoperatorios en presencia de un LQTS es evitar ambos tipos de drogas a menos que sea estrictamente necesario ${ }^{52,53,121}$.

Como puede apreciarse, el manejo perioperatorio de los pacientes con LQTS está lejos de ser consensuado, si bien existe acuerdo en líneas generales. La literatura es rica en revisiones y reportes de casos llevados a buen término, generando recomendaciones basadas en la experiencia en muchas oportunidades, y en otras, a la luz de datos obtenidos en estudios aislados. Aún no existe una forma veraz de objetivar el riesgo arritmogénico de una determinada droga, considerando el estado actual del conocimiento de la fisiopatología del LQTS, puesto que ni siquiera hay consenso en la forma correcta de evaluar la DRT. La mayoría de los estudios se ha limitado a evaluar su efecto sobre el QTc, lo cual, como se ha expuesto, no es sinónimo de arritmogenicidad, por lo que futuros estudios podrían refutar lo que hoy día es considerado como seguro o viceversa. Sin embargo, en virtud de la seguridad del paciente como primera motivación, y en el marco médico-legal que se vive en la actualidad, es la evidencia recopilada hasta ahora la que dictará la pauta por el momento.

\section{Referencias}

1. Davey PP. Which lead for Q-T interval measurements? Cardiology 2000;94(3):159-64.

2. Drew BJ, Califf RM, Funk M, Kaufman ES, Krucoff MW, Laks $\mathrm{MM}$ et al. American Heart Association; Councils on Cardiovascular Nursing, Clinical Cardiology, and Cardiovascular Disease in the Young. Practice standards for electrocardiographic monitoring in hospital settings: an American Heart Association scientific statement from the Councils on
Cardiovascular Nursing, Clinical Cardiology, and Cardiovascular Disease in the Young: endorsed by the International Society of Computerized Electrocardiology and the American Association of Critical-Care Nurses. Circulation 2004 Oct;110(17):2721-46.

3. Rautaharju PM, Surawicz B, Gettes LS, Bailey JJ, Childers

R, Deal BJ et al. American Heart Association Electrocardiography and Arrhythmias Committee, Council on Clinical Cardiology; American College of Cardiology Foundation; Heart Rhythm So- ciety; Endorsed by the International Society for Computerized Electrocardiology. AHA/ACCF/ HRS recommendations for the standardization and interpretation of the electrocardiogram: part IV: the ST segment, T and $\mathrm{U}$ waves, and the QT interval: a scientific statement from the American Heart Association Electrocardiography and Arrhythmias Committee, Council on Clinical Cardiology; the American College of Cardiology Foundation; and the Heart Rhythm Society. J Am Coll Cardiol 2009 
Mar;53(11):982-91.

4. Rautaharju PM, Zhou SH, Wong S, Calhoun HP, Berenson GS,

Prineas $\mathrm{R}$ et al. Sex differences in the evolution of the electrocardiographic QT interval with age. Can J Cardiol 1992 Sep;8(7):690-5.

5. Arslan E, Yiğiner O, Yavaşoğlu I, Ozçelik F, Kardeşoğlu E, Nalbant S. Effect of uncomplicated obesity on QT interval in young men. Pol Arch Med Wewn 2010 Jun;120(6):209-13.

6. Papaioannou A, Michaloudis D, Fraidakis O, Petrou A, Chaniotaki F, Kanoupakis E et al. Effects of weight loss on QT interval in morbidly obese patients. Obes Surg 2003 Dec;13(6):869-73.

7. Eberle T, Hessling G, Ulmer HE, Brockmeier K. Prediction of normal QT intervals in children. J Electrocardiol 1998;31 Suppl:121-5.

8. Rijnbeek PR, Witsenburg M, Schrama E, Hess J, Kors JA. New normal limits for the paediatric electrocardiogram. Eur Heart J 2001 Apr;22(8):702-11.

9. Cowan JC, Yusoff K, Moore M, Amos PA, Gold AE, Bourke JP et al. Importance of lead selection in QT interval measurement. Am J Cardiol 1988 Jan;61(1):837.

10. Faber TS, Kautzner J, Zehender M, Camm AJ, Malik M. Impact of electrocardiogram recording format on QT interval measurement and QT dispersion assessment. Pacing Clin Electrophysiol 2001 Dec;24(12):173947.

11. Murray A, McLaughlin NB, Bourke JP, Doig JC, Furniss SS, Campbell RW. Errors in manual measurement of QT intervals. Br Heart J 1994 Apr;71(4):386-90.

12. Luo S, Michler K, Johnston P, Macfarlane PW. A comparison of commonly used QT correction formulae: the effect of heart rate on the QTc of normal ECGs. J Electrocardiol 2004;37 Suppl:81-90.

13. Rautaharju PM, Zhang ZM. Linearly scaled, rate-invariant normal limits for QT interval: eight decades of incorrect application of power functions. $\mathrm{J}$ Cardiovasc Electrophysiol 2002 Dec;13(12):1211-8.

14. Antzelevitch C. Role of transmural dispersion of repolarization in the genesis of drug-induced torsades de pointes. Heart rhythm : the official journal of the Heart Rhythm Society. 2005;2(2 Suppl):S9-15. Epub 2005/10/29. 10.1016/j.hrthm.2004.09.011.

15. Shimizu W, McMahon B, Antzelevitch C. Sodium pentobarbital reduces transmural dispersion of repolarization and prevents torsades de Pointes in models of acquired and congenital long QT syndrome. J Cardiovasc Electrophysiol $1999 \mathrm{Feb}$;0(2):154-64.

16. Grimm W, Steder U, Menz V, Hoffmann J, Maisch B. Effect of amiodarone on QT dispersion in the 12-lead standard electrocardiogram and its significance for subsequent arrhythmic events. Clin Cardiol 1997 Feb;20(2):107-10.

17. Kautzner J, Malik M. QT interval dispersion and its clinical utility. Pacing Clin Electrophysiol 1997 Oct;20(10 Pt 2):2625-40.

18. Malik M, Batchvarov VN. Measurement, interpretation and clinical potential of QT dispersion. J Am Coll Cardiol 2000 Nov;36(6):1749-66.

19. Batchvarov V, Malik M. Measurement and interpretation of QT dispersion. Prog Cardiovasc Dis 2000 Mar-Apr;42(5):325-44.

20. Antzelevitch C, Sicouri S, Di Diego JM, Burashnikov A,
Viskin S, Shimizu W, et al. Does Tpeak-Tend provide an index of transmural dispersion of repolarization? Heart rhythm: the official journal of the Heart Rhythm Society. 2007;4(8):1114-6; author reply 6-9. Epub 2007/08/07. 10.1016/j. hrthm.2007.05.028.

21. Yamaguchi M, Shimizu M, Ino $\mathrm{H}$, Terai H, Uchiyama K, Oe $\mathrm{K}$ et al. $\mathrm{T}$ wave peak-to-end interval and QT dispersion in acquired long QT syndrome: a new index for arrhythmogenicity. Clin Sci (Lond) 2003 Dec;105(6):671-6.

22. Xia Y, Liang Y, Kongstad O, Liao Q, Holm M, Olsson B, et al. In vivo validation of the coincidence of the peak and end of the $\mathrm{T}$ wave with full repolarization of the epicardium and endocardium in swine. Heart rhythm : the official journal of the Heart Rhythm Society. 2005;2(2):1629. Epub 2005/04/27. 10.1016/j. hrthm.2004.11.011.

23. Gupta P, Patel C, Patel H, Narayanaswamy $\mathrm{S}$, Malhotra $\mathrm{B}$, Green JT, et al. T(p-e)/QT ratio as an index of arrhythmogenesis. J Electrocardiol 2008 NovDec;41(6):567-74.

24. Moss AJ. T-wave patterns associated with the hereditary long QT syndrome. Card Electrophysiol Rev 2002 Sep;6(3):311-5.

25. Alders M, Mannens M. RomanoWard Syndrome. In: Pagon RA, Adam MP, Bird TD, Dolan CR, Fong CT, Stephens K, editors. GeneReviews. Seattle (WA); 1993.

26. Tranebjaerg L, Samson RA, Green GE. Jervell and LangeNielsen Syndrome. In: Pagon RA, Adam MP, Bird TD, Dolan CR, Fong CT, Stephens K, editors. GeneReviews. Seattle (WA); 1993 
27. Crotti L, Celano G, Dagradi F, Schwartz PJ. Congenital long QT syndrome. Orphanet J Rare Dis 2008 Jul;3(1):18.

28. Modell SM, Lehmann MH. The long QT syndrome family of cardiac ion channelopathies: a HuGE review. Genetics in medicine : official journal of the American College of Medical Genetics. 2006;8(3):143-55. Epub 2006/03/17. 10.1097/01. gim.0000204468.85308.86.

29. Antzelevitch C. Ionic, molecular, and cellular bases of QT-interval prolongation and torsade de pointes. Europace : European pacing, arrhythmias, and cardiac electrophysiology: journal of the working groups on cardiac pacing, arrhythmias, and cardiac cellular electrophysiology of the European Society of Cardiology. 2007;9 Suppl 4:iv4-15. Epub 2007/11/21.

30. Camm AJ, Janse MJ, Roden DM, Rosen MR, Cinca J, Cobbe SM. Congenital and acquired long QT syndrome. Eur Heart J 2000 Aug;21(15):1232-7.

31. Kaufman ES. Mechanisms and clinical management of inherited channelopathies: long QT syndrome, Brugada syndrome, catecholaminergic polymorphic ventricular tachycardia, and short QT syndrome. Heart rhythm : the official journal of the Heart Rhythm Society. 2009;6(8 Suppl):S51-5. Epub 2009/08/06.

32. Schwartz PJ, Priori SG, Spazzolini C, Moss AJ, Vincent GM, Napolitano C, et al. Genotypephenotype correlation in the long-QT syndrome: gene-specific triggers for life-threatening arrhythmias. Circulation 2001 Jan;103(1):89-95.

33. Vincent GM, Schwartz PJ, Denjoy I, Swan H, Bithell C,
Spazzolini C et al. High efficacy of beta-blockers in long-QT syndrome type 1 : contribution of noncompliance and QTprolonging drugs to the occurrence of beta-blocker treatment "failures". Circulation 2009 Jan;119(2):215-21.

34. Shimizu W, Antzelevitch C. Differential effects of betaadrenergic agonists and antagonists in LQT1, LQT2 and LQT3 models of the long QT syndrome. J Am Coll Cardiol 2000 Mar;35(3):778-86.

35. Moss AJ, Zareba W, Hall WJ, Schwartz PJ, Crampton RS, Benhorin J, et al. Effectiveness and limitations of beta-blocker therapy in congenital long-QT syndrome. Circulation 2000 Feb;101(6):616-23.

36. Priori SG, Napolitano C, Schwartz PJ, Grillo M, Bloise R, Ronchetti E, et al. Association of long QT syndrome loci and cardiac events among patients treated with beta-blockers. JAMA 2004 Sep;292(11):1341-4.

37. Windle JR, Geletka RC, Moss AJ, Zareba W, Atkins DL. Normalization of ventricular repolarization with flecainide in long QT syndrome patients with SCN5A:DeltaKPQ mutation. Annals of noninvasive electrocardiology: the official journal of the International Society for Holter and Noninvasive Electrocardiology. Inc. 2001;6(2):153-8. Epub 2001 May 03.

38. Moss AJ, Windle JR, Hall WJ, Zareba W, Robinson JL, McNitt $\mathrm{S}$, et al. Safety and efficacy of flecainide in subjects with Long QT-3 syndrome (DeltaKPQ mutation): a randomized, doubleblind, placebo-controlled clinical trial. Annals of noninvasive electrocardiology: the official journal of the International Society for
Holter and Noninvasive Electrocardiology. Inc. 2005;10(4 Suppl):59-66. Epub 2005 Nov 09.

39. Schwartz PJ, Priori SG, Cerrone M, Spazzolini C, Odero A, Napolitano C, et al. Left cardiac sympathetic denervation in the management of high-risk patients affected by the long-QT syndrome. Circulation 2004 Apr;109(15):1826-33.

40. Etheridge SP, Sanatani S, Cohen MI, Albaro CA, Saarel EV, Bradley DJ. Long QT syndrome in children in the era of implantable defibrillators. J Am Coll Cardiol 2007 Oct;50(14):1335-40.

41. Schwartz PJ, Spazzolini C, Priori SG, Crotti L, Vicentini A, Landolina $\mathrm{M}$, et al. Who are the long-QT syndrome patients who receive an implantable cardioverter-defibrillator and what happens to them?: data from the European Long-QT Syndrome Implantable Cardioverter-Defibrillator (LQTS ICD) Registry. Circulation 2010 Sep;122(13):1272-82.

42. Schwartz PJ, Spazzolini C, Crotti L. All LQT3 patients need an ICD: true or false? Heart rhythm : the official journal of the Heart Rhythm Society. 2009;6(1):11320. Epub 2009/01/06. 10.1016/j. hrthm.2008.10.017.

43. Kallergis EM, Goudis CA, Simantirakis EN, Kochiadakis GE, Vardas PE. Mechanisms, risk factors, and management of acquired long QT syndrome: a comprehensive review. TheScientificWorldJournal. 2012;2012:212178. Epub 2012/05/18. 10.1100/2012/212178.

44. Kaye AD, Volpi-Abadie J, Bensler JM, Kaye AM, Diaz JH. QT interval abnormalities: risk factors and perioperative manage- 
ment in long QT syndromes and Torsades de Pointes. J Anesth 2013 Aug;27(4):575-87.

45. Charlton NP, Lawrence DT, Brady WJ, Kirk MA, Holstege CP. Termination of drug-induced torsades de pointes with overdrive pacing. Am J Emerg Med 2010 Jan;28(1):95-102.

46. Curry TB, Gaver R, White RD. Acquired long QT syndrome and elective anesthesia in children. Paediatr Anaesth 2006 Apr;16(4):471-8.

47. Khan IA. Clinical and therapeutic aspects of congenital and acquired long QT syndrome. Am J Med 2002 Jan;112(1):58-66.

48. Viskin S. Cardiac pacing in the long QT syndrome: review of available data and practical recommendations. J Cardiovasc Electrophysiol 2000 May;11(5):593-600.

49. Torsades de Pointes VS. Curr Treat Options Cardiovasc Med 1999;1(2):187-95.

50. Mandal B, Kaur G, Batra YK, Mahajan S. Manifestation of Long QT syndrome with normal QTc interval under anesthesia: a case report. Paediatr Anaesth 2011 Dec;21(12):1265-7.

51. Michaloudis DG, Kanakoudis FS, Xatzikraniotis A, Bischiniotis TS. The effects of midazolam followed by administration of either vecuronium or atracurium on the QT interval in humans. Eur J Anaesthesiol 1995 Nov;12(6):577-83.

52. Kies SJ, Pabelick CM, Hurley HA, White RD, Ackerman MJ. Anesthesia for patients with congenital long QT syndrome. Anesthesiology 2005 Jan;102(1):204-10.

53. Fazio G, Vernuccio F, Grutta G, Re GL. Drugs to be avoided in patients with long QT syndrome: focus on the anaesthesiological management. World J Cardiol 2013 Apr;5(4):87-93.

54. Nishiyama N, Sato T, Aizawa Y, Nakagawa S, Kanki H. Extreme QT prolongation during therapeutic hypothermia after cardiac arrest due to long QT syndrome. The American journal of emergency medicine. 2012;30(4):638 e5-8. Epub 2011/04/05. 10.1016/j.ajem.2011.02.019.

55. Nagatomo T, Fan Z, Ye B, Tonkovich GS, January CT, Kyle JW, et al. Temperature dependence of early and late currents in human cardiac wild-type and long Q-T DeltaKPQ Na+ channels. Am J Physiol 1998 Dec;275(6 Pt 2):H2016-24.

56. Homme JH, White RD, Ackerman MJ. Management of ventricular fibrillation or unstable ventricular tachycardia in patients with congenital long-QT syndrome: a suggested modification to ACLS guidelines. Resuscitation 2003 Oct;59(1):1115.

57. Johnston J, Pal S, Nagele P. Perioperative torsade de pointes: a systematic review of published case reports. Anesth Analg 2013 Sep;117(3):559-64.

58. Owczuk R, Wujtewicz MA, Zienciuk-Krajka A, LasińskaKowara M, Piankowski A, Wujtewicz M. The influence of anesthesia on cardiac repolarization. Minerva Anestesiol 2012 Apr;78(4):483-95.

59. Owczuk R, Wujtewicz MA, Sawicka W, Piankowski A, Polak-Krzeminska A, Morzuch $\mathrm{E}$ et al. The effect of intravenous lidocaine on QT changes during tracheal intubation. Anaesthesia 2008 Sep;63(9):924-31.

60. Hume-Smith HV, Sanatani S, Lim J, Chau A, Whyte SD. The effect of propofol concentration on dispersion of myocardial re- polarization in children. Anesth Analg 2008 Sep;107(3):806-10.

61. Kazanci D, Unver S, Karadeniz U, Iyican D, Koruk S, Yilmaz $\mathrm{MB}$, et al. A comparison of the effects of desflurane, sevoflurane and propofol on QT, QTc, and P dispersion on ECG. Ann Card Anaesth 2009 JulDec;12(2):107-12.

62. Whyte SD, Booker PD, Buckley DG. The effects of propofol and sevoflurane on the QT interval and transmural dispersion of repolarization in children. Anesth Analg 2005 Jan;100(1):71-7.

63. Kleinsasser A, Kuenszberg E, Loeckinger A, Keller C, Hoermann $\mathrm{C}$, Lindner $\mathrm{KH}$, et al. Sevoflurane, but not propofol, significantly prolongs the Q-T interval. Anesth Analg 2000 Jan;90(1):25-7.

64. Kleinsasser A, Loeckinger A, Lindner KH, Keller C, Boehler $\mathrm{M}$, Puehringer F. Reversing sevoflurane-associated Q-Tc prolongation by changing to propofol. Anaesthesia 2001 Mar;56(3):248-50.

65. Erdil F, Demirbilek S, Begec Z, Ozturk E, Ersoy MO. Effects of propofol or etomidate on QT interval during electroconvulsive therapy. J ECT 2009 Sep;25(3):174-7.

66. Lischke V, Wilke HJ, Probst S, Behne M, Kessler P. Prolongation of the QT-interval during induction of anesthesia in patients with coronary artery disease. Acta Anaesthesiol Scand 1994 Feb;38(2):144-8.

67. Cafiero T, Di Minno RM, Di Iorio C. QT interval and QT dispersion during the induction of anesthesia and tracheal intubation: a comparison of remifentanil and fentanyl. Minerva Anestesiol 2011 Feb;77(2):1605. 
68. Chang DJ, Kweon TD, Nam SB, Lee JS, Shin CS, Park CH, et al. Effects of fentanyl pretreatment on the QTc interval during propofol induction. Anaesthesia 2008 Oct;63(10):1056-60.

69. Blair JR, Pruett JK, Crumrine RS, Balser JJ. Prolongation of QT interval in association with the administration of large doses of opiates. Anesthesiology 1987 Sep;67(3):442-3.

70. Tsutsui K, Hayami N, Kunishima T, Sugiura A, Mikamo T, Kanamori K, et al. Dexmedetomidine and clonidine inhibit ventricular tachyarrhythmias in a rabbit model of acquired long QT syndrome. Circulation journal : official journal of the Japanese Circulation Society. 2012;76(10):2343-7. Epub 2012/07/13. 10.1253/circj.CJ-120171.

71. Burns KM, Greene EA, Long QT. Syndrome Unmasked by Dexmedetomidine: A Case Report. Congenit Heart Dis 2013.

72. Michaloudis DG, Kanakoudis FS, Petrou AM, Konstantinidou AS, Pollard BJ. The effects of midazolam or propofol followed by suxamethonium on the QT interval in humans. Eur J Anaesthesiol 1996 Jul;13(4):364-8.

73. Annila P, Yli-Hankala A, Lindgren L. Effect of atropine on the QT interval and T-wave amplitude in healthy volunteers. Br J Anaesth 1993 Nov;71(5):736-7.

74. Beccaria E, Brun S, Gaita F, Giustetto C, Conti M. [Torsade de pointes during an atropine sulfate test in a patient with congenital long QT syndrome]. Cardiologia 1989 Dec;34(12):103943.

75. Saarnivaara L, Simola M. Effects of four anticholinesteraseanticholinergic combinations and tracheal extubation on QTc interval of the ECG, heart rate and arterial pressure. Acta Anaesthesiol Scand 1998 Apr;42(4):460-3.

76. Shields JA. Heart block and prolonged Q-Tc interval following muscle relaxant reversal: a case report. AANA J 2008 Feb;76(1):41-5.

77. Pleym H 1, Bathen J 2, Spigset O 3, Gisvold SE 1. Ventricular fibrillation related to reversal of the neuromuscular blockade in a patient with long QT syndrome. Acta Anaesthesiol Scand 1999 Mar;43(3):352-5.

78. Plaud B, Meretoja O, Hofmockel R, Raft J, Stoddart PA, van Kuijk $\mathrm{JH}$, et al. Reversal of rocuronium-induced neuromuscular blockade with sugammadex in pediatric and adult surgical patients. Anesthesiology 2009 Feb;110(2):284-94.

79. de Kam PJ, van Kuijk J, Prohn M, Thomsen T, Peeters P. Effects of sugammadex doses up to 32 $\mathrm{mg} / \mathrm{kg}$ alone or in combination with rocuronium or vecuronium on QTc prolongation: a thorough QTc study. Clin Drug Investig 2010;30(9):599-611.

80. de Kam PJ, van Kuijk J, Smeets J, Thomsen T, Peeters P. Sugammadex is not associated with QT/ QTc prolongation: methodology aspects of an intravenous moxifloxacin-controlled thorough QT study. Int J Clin Pharmacol Ther 2012 Aug;50(8):595-604.

81. Riley RH, Song JW, Paul VE, Powell A. Safe use of sugammadex in long QT syndrome. Anaesth Intensive Care 2010 Nov;38(6):1138-9.

82. Hüneke R, Fassl J, Rossaint R, Lückhoff A. Effects of volatile anesthetics on cardiac ion channels. Acta Anaesthesiol Scand 2004 May;48(5):547-61.

83. Schmeling WT, Warltier DC,
McDonald DJ, Madsen KE, Atlee JL, Kampine JP. Prolongation of the QT interval by enflurane, isoflurane, and halothane in humans. Anesth Analg 1991 Feb;72(2):137-44.

84. Karagöz AH, Basgul E, Celiker $\mathrm{V}$, Aypar U. The effect of inhalational anaesthetics on QTc interval. Eur J Anaesthesiol 2005 Mar;22(3):171-4.

85. Whyte SD, Sanatani S, Lim J, Booker PD. A comparison of the effect on dispersion of repolarization of age-adjusted MAC values of sevoflurane in children. Anesth Analg 2007 Feb;104(2):277-82.

86. Kim HS, Kim JT, Kim CS, Kim SD, Kim K, Yum MK. Effects of sevoflurane on QT parameters in children with congenital sensorineural hearing loss. Anaesthesia 2009 Jan;64(1):3-8.

87. Saussine M, Massad I, Raczka F, Davy JM, Frapier JM. Torsade de pointes during sevoflurane anesthesia in a child with congenital long QT syndrome. Paediatr Anaesth 2006 Jan;16(1):63-5.

88. Owczuk R, Wujtewicz MA, Sawicka W, Lasek J, Wujtewicz $M$. The Influence of desflurane on QTc interval. Anesthesia and analgesia. 2005;101(2):41922, table of contents. Epub 2005/07/23. 10.1213/01. ANE.0000154198.41162.FA.

89. Silay E, Kati I, Tekin M, Guler $\mathrm{N}$, Huseyinoglu UA, Coskuner I et al. Comparison of the effects of desflurane and sevoflurane on the QTc interval and QT dispersion. Acta Cardiol 2005 Oct;60(5):459-64.

90. Gürkan Y, Canatay H, Agacdiken A, Ural E, Toker K. Effects of halothane and sevoflurane on QT dispersion in paediatric patients. Paediatr Anaesth 2003 Mar;13(3):223-7. 
91. Nathan AT, Berkowitz DH, Montenegro LM, Nicolson SC, Vetter VL, Jobes DR. Implications of anesthesia in children with long QT syndrome. Anesth Analg 2011 May;112(5):1163-8.

92. Owczuk R, Sawicka W, Wujtewicz MA, Kawecka A, Lasek J, Wujtewicz M. Influence of spinal anesthesia on corrected QT interval. Reg Anesth Pain Med 2005 Nov-Dec;30(6):548-52.

93. Al-Refai A, Gunka V, Douglas J. Spinal anesthesia for Cesarean section in a parturient with long QT syndrome. Canadian journal of anaesthesia $=$ Journal canadien d'anesthesie. 2004;51(10):9936. Epub 2004/12/03. 10.1007/ BF03018486.

94. Sen S, Ozmert G, Turan H, Caliskan E, Onbasili A, Kaya D. The effects of spinal anesthesia on QT interval in preeclamptic patients. Anesth Analg 2006 Nov;103(5):1250-5.

95. Behl S, Wauchob TD. Long QT syndrome: anaesthetic management at delivery. Int J Obstet Anesth 2005 Oct;14(4):347-50.

96. Hodgkinson P, McAtamney D. Combined spinal-epidural anaesthesia for caesarean section in a patient with long QT syndrome. Int J Obstet Anesth 2008 Jan;17(1):78-80.

97. Ganta R, Roberts C, Elwood RJ, Maddineni VR. Epidural anesthesia for cesarean section in a patient with Romano-Ward syndrome. Anesth Analg 1995 Aug;81(2):425-6.

98. Minakami H, Nakayama T, Ohno T, Kuroki S, Sato I. Effect of vaginal delivery on the Q-Tc interval in a patient with the long Q-T (Romano-Ward) syndrome. J Obstet Gynaecol Res 1999 Aug;25(4):251-4.

99. Ryan H. Anaesthesia for cae- sarean section in a patient with Jervell, Lange-Nielsen syndrome. Canadian journal of anaesthesia $=$ Journal canadien d'anesthesie. 1988;35(4):422-4. Epub 1988/07/01.

100. Charbit B, Funck-Brentano C, Samain E, Jannier-Guillou V, Albaladejo P, Marty J. QT interval prolongation after oxytocin bolus during surgical induced abortion. Clin Pharmacol Ther 2004 Oct;76(4):359-64.

101. Bruyere M, Ait Hamou N, Benhamou D, Chousterman B, Boulard V, Charbit B. QT interval prolongation following carbetocin in prevention of postcesarean delivery hemorrhage. Int J Obstet Anesth 2013.

102. Guillon A, Leyre S, Remérand F, Taihlan B, Perrotin F, Fusciardi J, et al. Modification of Tp-e and QTc intervals during caesarean section under spinal anaesthesia. Anaesthesia 2010 Apr;65(4):337-42.

103. Charbit B, Funck-Brentano C, Benhamou D, Weissenburger J. Effects of oxytocin on Purkinje fibres. Br J Anaesth 2012 Jun;108(6):1039-41.

104. Sun ZH, Swan H, Viitasalo M, Toivonen L. Effects of epinephrine and phenylephrine on QT interval dispersion in congenital long QT syndrome. J Am Coll Cardiol 1998 May;31(6):1400-5.

105. Drake E, Preston R, Douglas J. Brief review: anesthetic implications of long QT syndrome in pregnancy. Canadian journal of anaesthesia $=$ Journal canadien d'anesthesie. 2007;54(7):56172. Epub 2007/07/03. 10.1007/ BF03022321.

106. Mitsutake A, Takeshita A, Kuroiwa A, Nakamura M. Usefulness of the Valsalva maneuver in management of the long QT syndrome. Circulation 1981
May;63(5):1029-35.

107. Di Iorio C, Cafiero T, Di Minno RM. The effects of pneumoperitoneum and head-up position on heart rate variability and QT interval dispersion during laparoscopic cholecystectomy. Minerva Anestesiol 2010 Nov;76(11):882-9.

108. Pimentel L, Mayo D. Chronic methadone therapy complicated by torsades de pointes: a case report. J Emerg Med 2008 Apr;34(3):287-90.

109. Kuryshev YA, Bruening-Wright A, Brown AM, Kirsch GE. Increased cardiac risk in concomitant methadone and diazepam treatment: pharmacodynamic interactions in cardiac ion channels. J Cardiovasc Pharmacol 2010 Oct;56(4):420-30.

110. Russell L, Levine D. Methadoneinduced Torsades de pointes. R I Med J (2013). 2013;96(8):20-1. Epub 2013/08/08.

111. Song MK, Bae EJ, Baek JS, Kwon BS, Kim GB, Noh CI, et al. QT Prolongation and Life Threatening Ventricular Tachycardia in a Patient Injected With Intravenous Meperidine (Demerol ${ }^{\circledR}$ ). Korean Circ J 2011 Jun;41(6):342-5.

112. Habib AS, Gan TJ. Food and drug administration black box warning on the perioperative use of droperidol: a review of the cases. Anesth Analg 2003 May;96(5):1377-9.

113. Nuttall GA, Malone AM, Michels CA, Trudell LC, Renk TD, Marienau ME, et al. Does low-dose droperidol increase the risk of polymorphic ventricular tachycardia or death in the surgical patient? Anesthesiology 2013 Feb;118(2):382-6.

114. Dershwitz M. There should be a threshold dose for the FDA black-box warning on 
droperidol. Anesth Analg 2003 Nov;97(5):1542-3.

115. Charbit B, Alvarez JC, Dasque E, Abe E, Démolis JL, FunckBrentano C. Droperidol and ondansetron-induced QT interval prolongation: a clinical drug interaction study. Anesthesiology 2008 Aug;109(2):206-12.

116. Kuryshev YA, Brown AM, Wang L, Benedict CR, Rampe D. Interactions of the 5-hydroxytryptamine 3 antagonist class of antiemetic drugs with human cardiac ion channels. J Pharmacol Exp Ther 2000 Nov;295(2):614-20.

117. Charbit B, Albaladejo P, FunckBrentano C, Legrand M, Samain E, Marty J. Prolongation of
QTc interval after postoperative nausea and vomiting treatment by droperidol or ondansetron. Anesthesiology 2005 Jun;102(6):1094-100.

118. Fortney JT, Gan TJ, Graczyk S, Wetchler B, Melson T, Khalil $\mathrm{S}$, et al. A comparison of the efficacy, safety, and patient satisfaction of ondansetron versus droperidol as antiemetics for elective outpatient surgical procedures. S3A-409 and S3A-410 Study Groups. Anesth Analg 1998 Apr;86(4):731-8.

119. Jackson CW, Sheehan AH, Reddan JG. Evidence-based review of the black-box warning for droperidol. American journal of health-system phar- macy: AJHP: official journal of the American Society of Health-System Pharmacists. 2007;64(11):1174-86. Epub 2007/05/24. 10.2146/ ajhp060505.

120. Gan TJ, Meyer T, Apfel CC, Chung F, Davis PJ, Eubanks S, et al. Consensus guidelines for managing postoperative nausea and vomiting. Anesthesia and analgesia. 2003;97(1):6271 , table of contents. Epub 2003/06/24. 10.1213/01. ANE.0000068580.00245.95.

121. Staikou C, Chondrogiannis K, Mani A. Perioperative management of hereditary arrhythmogenic syndromes. Br J Anaesth 2012 May;108(5):730-44. 